\title{
El médico: su satisfacción como paciente o como tratante de otro médico. Comparación con la población general en Venezuela: estudio piloto.
}

\author{
Trino Baptista ${ }^{1}$,Heidy Delgado ${ }^{2}$ Ricardo Salcedo ${ }^{2}$ Euderruh Uscátegui ${ }^{2}$ \\ y Ana Serrano ${ }^{2}$ \\ ${ }^{1}$ Departamento de Fisiología, Facultad de Medicina, Universidad de Los Andes, \\ Mérida, Venezuela. \\ ${ }^{2}$ Unidad de Psiquiatría, Instituto Autónomo Hospital Universitario de Los Andes, \\ Mérida, Venezuela.
}

Palabras clave: médico como paciente; médico tratante de otro médico; nivel de satisfacción con la atención médica.

Resumen. La forma como el médico aborda su salud, la de su familia y la de los colegas a los que atiende es un tema de interés práctico y humanístico. Sin embargó, las publicaciones sobre este tema son escasas y la discusión se basa a menudo en información anecdótica. En el presente estudio se determinó en Mérida, Venezuela, el grado de satisfacción de los médicos con la atención que han recibido como pacientes, y se comparó en forma concurrente con la de sujetos de la población general (PG). Se evaluó igualmente el nivel de satisfacción de los médicos en su condición de profesionales tratantes de otro colega. La hipótesis de trabajo fue que un porcentaje significativo de médicos tiene una opinión negativa, influida por su edad y sexo. La variable objeto de estudio se analizó en tres niveles: 1) opinión positiva o satisfacción; 2) opinión neutra, y 3) opinión negativa o insatisfacción. El mareo temporal fue el nivel de satisfacción/insatisfacción 'en general', y la 'última vez' que se brindó o se recibió asistencia profesional. Se seleccionaron en forma probabilística 52 médicos especialistas, 84 médicos residentes y 494 sujetos de la PG. Se utilizó una encuesta autoadministrada cuyo análisis psicométrico demostró un coeficiente de validez de contenido de 0,82 , el cual califica como 'bueno'. Los especialistas masculinos reportaron las frecuencias más elevadas de insatisfacción con el trato recibido de sus pacientes médicos $(28,6 \%, \mathrm{p}=0,05-0,1)$ y en su desempeño profesional con los colegas $(7,1 \%, \mathrm{p}>0,05)$. De igual forma, los especialistas masculinos presentaron las frecuencias más elevadas de insatisfacción como pacientes $(38,4-42,9 \%, \mathrm{p}<0,05)$. Las residentes reportaron los niveles más ba-

Autor de correspondencia: Trino Baptista. Departamento de Fisiología, Facultad de Medicina, Av. Tulio Febres Cordero, Mérida, 5101-A, Venezuela, Tlf: +58 4247447 318. Correo electrónico: trinbap@yahoo.com 
jos de insatisfacción como pacientes o tratantes entre los médicos, aunque los valores no alcanzaron significación estadística. La PG masculina reportó valores significativamente más bajos de insatisfacción que todos los médicos $(6,6 \%, p$ $<0,05)$. En conclusión, una frecuencia significativa de médicos, particularmente los hombres especialistas, está insatisfecha tanto como tratante de otros médicos, como en su rol de paciente. Deben diseñarse programas de educación profesional ad hoc para abordar esta importante dinámica asistencial, la cual

\title{
Doctors: their satisfaction as patients or when treating another doctor. Comparison with the general population in Venezuela: a pilot study.
}

\author{
Invest Clin 2021; 62 (4): 339-356
}

Key words: doctors as patients; doctors treating other doctors; level of satisfaction with medical attention.

\begin{abstract}
The way in which physicians manage their health and that of their families and colleagues is an issue of practical and humanistic value. However, there are few published studies on these topies, and discussion is often based on anecdotes. We determined the level of satisfaction of physicians when treating a colleague and, in turn, when they became patients in Mérida, Venezuela, We additionally, evaluated the satisfaction with the medical attention in subjects from the general population (PG). The variable under study was analyzed in three levels: 1) positive opinion or satisfaction; 2) neutral opinion and 3) negative opinion or dissatisfaction. The answers' temporal frame referred to the opinion 'in general' and in 'the last occasion' that the respondents referred when provided or received medical assistance. We obtained a probabilistic sample of $\mathbf{5 2}$ specialists and 84 residents, and 494 subjects from the GP. All subjects answered a self-administered questionnaire, whose psychometric assessment showed a content validity coefficient of 0.82 , which qualifies as 'good'. All the subjects reported their satisfaction as patients, and the physicians also reported their satisfaction level when treating other physicians. Male specialists reported the highest dissatisfaction frequencies in the personal attitudes toward them, of the physicians that they treated $(28.6 \%, \mathrm{p}=0.05-0.1)$ and their performance as treating physicians $(7.1 \%, \mathrm{p}>0.05)$. Similarly, the male specialists $(38.4-42.9 \%, \mathrm{p}<0.05)$ reported the highest dissatisfaction frequencies as patients. Female residents, compared to all the physicians, reported the lowest dissatisfaction levels as providers or recipient of medical attention, but the figures did not reach statistical significance. The GP masculine group reported lower dissatisfaction levels than the physicians as a group $(6.6 \%, \mathrm{p}<0.05)$. We conclude that a significant proportion of physicians, particularly male specialists, are dissatisfied in their roles of treating physicians of another colleague and are also dissatisfied as patients. Ad hoc programs of professional education should be designed to modify this important health issue, which could be even more important during the current COVID-19 pandemic.
\end{abstract}

Recibido: 24-03-2021 Aceptado: 03-09-2021 


\section{INTRODUCCIÓN}

El cuidado deficiente de la salud del médico es un tema central en la profesión. Albujar-Baca (1), especula que a menudo los médicos creen tener una salud invulnerable, subestiman los síntomas que presentan, optan por la automedicación, y, si consultan, lo hacen de manera informal, recurren a argumentos sin base racional para explicar las causas de su dolencia, aceptan tratamientos no probados científicamente, niegan su enfermedad, se quejan de la falta de consideración y delicadeza de sus médicos tratantes, etc. Esta actitud de "autosuficiencia" puede explicar por qué pocos galenos tienen un médico de cabecera (2). En un sentido anecdótico o informal, también se afirma que el médico y sus familiares, no son atendidos en forma óptima por sus colegas, y/o manifiestan actitudes que dificultan su tratamiento.

En el contexto competitivo de la atención sanitaria organizada alrededor de empresas aseguradoras, las investigaciones se han centrado en la satisfacción de los usuarios (3), y podrían descuidar el justo bienestar del médico y revertirse contra la calidad de la atención (4). El imperativo de cuidar la salud del médico se refuerza por los hallazgos de que la prevalencia de "burnout" entre los galenos sobrepasa el 50\% (5), y la tasa de suicidio excede significativamente a la de la población general (PG) (6).

Dado que este tema ha sido poco estudiado en Hispanoamérica, la presente investigación planteó como objetivos evaluar la opinión y satisfacción de médicos especialistas y residentes sobre su labor como tratantes de otros médicos, y como pacientes. Se utilizó como grupo de comparación la opinión/satisfacción como pacientes en sujetos de la PG. La hipótesis de trabajo fue que una proporción significativa de médicos tiene una opinión negativa de su rol como paciente y como tratante de otro galeno. En el marco de esta hipótesis la interrogante específica que se planteó fue si la edad y el sexo de los galenos influyen en su percepción de la calidad de la atención médica recibida u ofrecida a otros colegas. Se exploró igualmente la influencia de otras variables explicativas como la especialidad médica y el tipo de relación social además de la profesional.

\section{MATERIALES Y MÉTODOS}

La investigáción se realizó en Mérida, Venezuela, bajo la aprobación del Comité de Ética del Hospital Universitario de los Andes (IAHULA). Se trató de un estudio transversal, observacional, de comparación entre grupos, de carácter cuantitativo y semicuantitativo.

\section{Sujetos, universo y muestra}

El universo fue la totalidad de los médicos del IAHULA, y la PG adulta (> 18 años) del Municipio Libertador de la ciudad de Mérida. Se obtuvo una muestra probabilística, estratificada por sexo y edad para la PG con base en el censo poblacional del año 2011. La muestra de los médicos fue estratificada por nivel profesional (especialista o residente) y sexo, y se obtuvo de una lista organizada por orden alfabético a través de selección sistemática mediante intervalos de 4 sujetos para los especialistas y de 7 sujetos para los residentes (7). Cuando un sujeto seleccionado de la lista no correspondía al sexo requerido, se procedía a seleccionar al siguiente sujeto. El tamaño de las muestras se calculó con los siguientes factores: $(8,9)$

$$
Z=1,96 ; \mathrm{p}=0,5 ; \mathrm{q}==0,5 ; \text { Error de }
$$
estimación $=0,1$.

\section{Criterios de inclusión/exclusión}

1. Trabajar como médico especialista o residente en el Instituto Autónomo Hospital Universitario de los Andes (I.A.H.U.L.A).

2. Previa explicación de los objetivos, participar voluntariamente en el estudio y brindar consentimiento informado verbal.

3. Los sujetos de la PG debían ser residentes en la ciudad durante el último año. 


\section{Procedimiento}

Los médicos contestaron la encuesta en su hogar, y luego la depositaron en alguno de varios recolectores dispuestos en la institución y debidamente identificados. La PG fue entrevistada por médicos residentes de Psiquiatría, en tres Centros Comerciales de todos los estratos económicos. La encuesta fue estrictamente anónima y el consentimiento informado fue verbal. Cada encuestador debía completar una cuota de sujetos tabulados por sexo y edad. A fin de optimizar la aleatorización, el entrevistador debidamente identificado, seleccionaba el tercer sujeto interpelado en forma sucesiva, le explicaba los objetivos del estudio, y solicitaba su participación voluntaria.

\section{Instrumento}

La encuesta fue diseñada por los investigadores de la presente investigación. Un estudio piloto determinó su comprensibilidad; su coeficiente de Validez de Contenido mediante Juicio de cuatro Expertos fue 0,82 (Bueno), calculado mediante la siguiente fórmula (10):

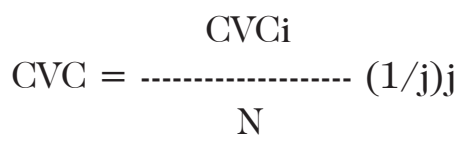

donde:

$(\mathrm{CVC}) \mathrm{i}=$ coeficiente de validez de contenido para cada ítem.

$\mathrm{N}=$ número de preguntas.

$\mathrm{j}=$ número de expertos.

El instrumento con repuestas tipo Likert (10) comprendía las siguientes secciones (Material suplementario 1 que puede ser solicitado al autor):

1. Preguntas 1-13: el médico como tratante de otro galeno.

2. Preguntas 14-26: los médicos y la PG como pacientes.

3. Las preguntas 13 y 26 fueron abiertas.
Las preguntas podían referirse a la experiencia como proveedor o usuario de atención médica 'en general' o 'la última vez' que lo requirió, dependiendo de la sección específica de la encuesta.

\section{Hipótesis}

Un porcentaje significativo de médicos tiene una opinión negativa de la atención médica que ha recibido a lo largo de su vida y la última vez que la necesitó. En forma similar, un porcentaje significativo de médicos tiene una opinión negativa de su experiencia como tratante de otros colegas. La distribución de frecuencia está influenciada por la edad y el sexo.

En forma adicional se incluyó la especialidad -clínica o quirúrgíca- del médico tratante y del médico-paciente en la última consulta y el tipo de relación social entre ellos (amigo o familia), como variables intervinientes a ser exploradas.

\section{Variables}

Variable objeto de estudio: nivel de satisfacción sobre la atención médica brindada (por los médicos) y recibida (todos los sujetos).

Variables explicativas: edad y sexo de los participantes, especialidad del médico tratante y del médico-paciente, y tipo de relación social entre ellos. La edad se tabuló en 4 grupos etarios: 20-29 años; 30-39 años; 40-49 años $\mathrm{y}>50$ años.

\section{Análisis estadístico}

Se utilizó el programa SPSS IBM 20. El instrumento se subdividió en módulos con base en las características de las preguntas, y se realizó un análisis factorial en cada módulo. Dado que las opiniones como proveedor de servicio o como usuario 'en general' y 'la última vez' fueron idénticas $(\mathrm{p} \cong 1$ ), las respuestas se agruparon dentro de cada módulo, y se consideraron como factores.

Utilizamos la prueba de chí cuadrado en cada factor y en preguntas no factorizadas. Escogimos un error típico de \pm 3 como 
punto de corte en los residuos tipificados corregidos (RTC), con un valor alfa $\leq 0,05$. Un RTC de 2,5-3, se consideró marǵinalmente significativo ( $\mathrm{p}$ entre 0,05 y 0,1 ). Si la Tabla de Contingencia de 2 x 2 celdas contenía más del $25 \%$ con $n<5$, utilizamos la prueba exacta de Fisher. A fin de controlar el error alfa, en los análisis con las Tablas de Contingencia de 2 x 2 , se utilizó la corrección de continuidad, y en las tablas con un mayor número de casillas se aplicó el método de Bonferroni para múltiples comparaciones.

Dado el elevado número de celdas con valores esperados $<5$, las respuestas se agruparon de la siguiente forma:

1. Preguntas con escala Likert de 5 ítems:
a. Polo positivo o satisfacción.
b. Neutro.
c. Polo negativo o insatisfacción.

4. Preguntas con escala Likert de 4 ítems:
a. Polo positivo o satisfacción.
b. Polo negativo o insatisfacción.

Se aplicío la prueba de Kendall's taub$b$ para evaluar la correlación entre la edad (por grupos etarios) y las puntuaciones en los factores y preguntas.

Las respuestas abiertas a las preguntas 13 y 26 se agruparon con base en su sentido, y sólo se presenta la distribución de frecuencias sin análisis inferencial de aquellas respuestas con una frecuencia $\geq 10 \%$.

La Fig. 1 describe el flujo de preguntas del instrumento y la organización modular del mismo.

\section{RESULTADOS}

El universo muestral fue: 205 médicos especialistas, 416 médicos residentes, y 49.813 sujetos de PG. La muestra calculada fue de 52 especialistas (25\% del total) y 59 residentes (14\% del total) con distribución de sexo de 1:1. En previsión de una baja tasa de respuesta, proporcionamos la encuesta a un número mayor de médicos que el calculado (35\% para especialistas y residentes). El porcentaje de respuestas fue de $100 \%$ y $142 \%$ respectivamente (Tabla I). La muestra calculada de la PG como grupo de comparación fue de 390 sujetos, sin embargo, decidimos evaluar 500 sujetos para optimizar la estadística inferencial. El porcentaje de respuesta de la PG fue de 99,1\%.

Las muestras de especialistas y residentes cumplieron con la distribución del sexo en el universo muestral. La muestra de la PG incluyó un $14 \%$ más de hombres que lo calculado.

Todos los análisis factoriales arrojaron valores de la Medida de Kaiser-Meyer-Olkin $=0,72-0,75$ y la prueba de esfericidad, $\mathrm{p}<$ 0,001 ; la magnitud del efecto fue pequeña ( $\mathrm{V}$ de Cramer entre 0,1-0,3) en todas las comparaciones.

\section{El médico tratando a otro médico (preguntas 1-13)}

Factor 1, preguntas 1-4 integradas: Actitud del médico paciente hacia el médico tratante y hacia los diagnósticos y tratamientos recibidos (en general y en la última consulta) (Tabla II).

Los especialistas masculinos presentaron la mayor frecuencia de opinión negativa sobre la actitud del médico-paciente, y los residentes femeninos, la menor frecuencia; la significación estadística fue marginal (RTC entre 2,5 y 3, p = 0,05-0,1). En conjunto, los médicos masculinos presentaron una frecuencia de opinión negativa significativamente mayor: $25,4 \%$ vs $15,1 \%, \mathrm{p}<0,05$.

No se observó diferencia significativa en la frecuencia de opinión negativa entre todos los especialistas vs todos los residentes: $26,9 \%$ vs. $25 \%, \mathrm{p}>0,05$.

Detectamos una correlación significativa positiva entre la edad y las puntuaciones en el factor 1, solamente en los especialistas masculinos $(\mathrm{r}$ [112] $=0,19, \mathrm{p}=0,025)$, es decir, a mayor edad, más negativa la opinión. 
Fhijogrann del extritis

\section{El médico tratando otro colę̧a médico}

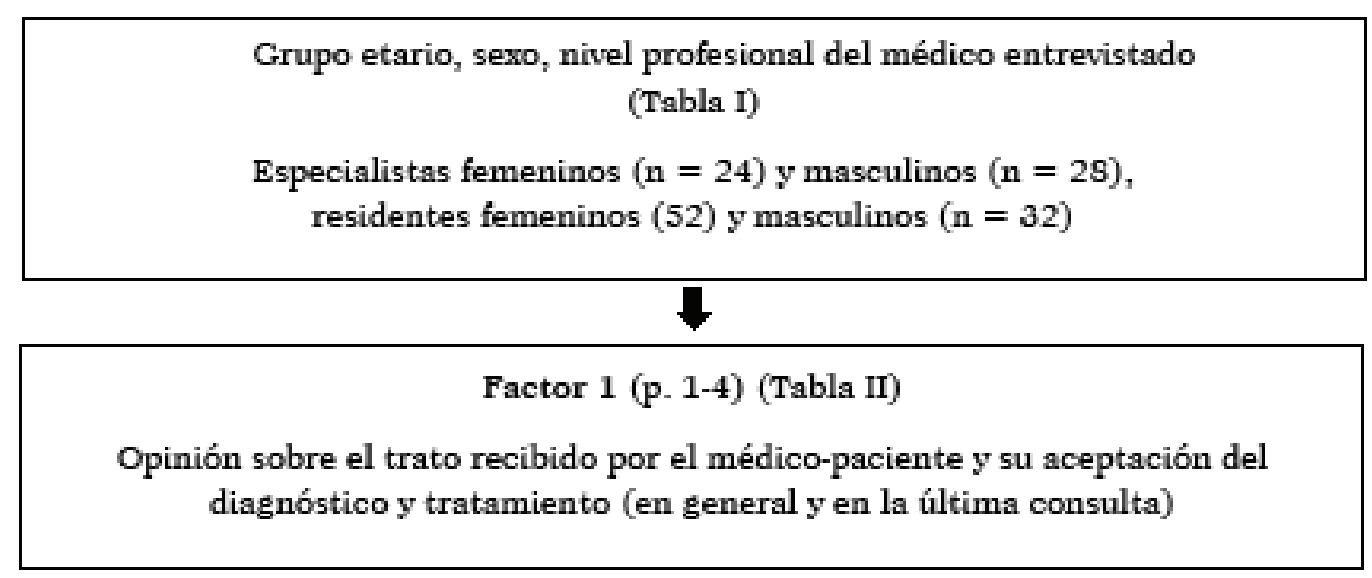

La última vez que atendió un colę̧a

\section{El médico-paciente}

Especialidad (p.5), sexo (p.6), tipo de relación social con el médico tratante (p.7)

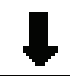

Factor 2 (p. 8-9) (Tabla II)

Opinión sobre el tiempo que le dedicó al colega paciente y sobre la información que le brindó sobre el diagnóstico y tratamiento

Interés por parte del médico-paciente hacia el tratamiento indicado (p. 10), contacto posterior con el colega-paciente (p. 11-12), descripción narrativa de la opinión sobre el médico como paciente (p. 13)

Fig. 1. Diseño del estudio y flujo de la entrevista. El médico como profesional tratante de otro médico (A). El médico y sujetos de la población general (PG) como pacientes (B). 
Los médicos y la población ǵeneral (PG) como pacientes

Especialistas femeninos $(n=24)$ y masculinos $(n=28)$ residentes femeninos

(52) y masculinos ( $n=32)$, PG femenina $(n=212)$, PG masculina $(p=282)$

(Tabla I)

\begin{tabular}{|c|}
\hline Factor 3 (p. 14-17) \\
Opinión sobre el trato brindado por el médico tratante y del diagnóstico y \\
tratamiento que recibió (en general y en la última consulta)
\end{tabular}

La última vez que fue a una consulta médica

El médico tratante

Especialidad (p.18), sexo (p.19), tipo de relación social con el médico tratante (p.20).

Factor 4 (p. 21-23)

Opinión sobre el tiempo que le dedicó el médico, sobre la información que le brindó sobre el diagnóstico y tratamiento y el seguimiento de su caso. Posibilidad de contacto con el médico tratante (p. 24-25). Descripción narrativa de la opinión sobre el médico tratante (p. 26)

Fig. 1. CONTINUACIÓN. Diseño del estudio y flujo de la entrevista. El médico como profesional tratante de otro médico (A). El médico y sujetos de la población general (PG) como pacientes (B). 


\section{TABLA I}

CARACTERÍSTICAS DEMOGRÁFICAS DE LOS MÉDICOS Y DE LA POBLACIÓN GENERAL.

\begin{tabular}{cccccc}
\hline \multicolumn{2}{c}{ Sujetos Sexo } & \multicolumn{4}{c}{ Grupo etario en años: $\mathrm{n}(\%)$} \\
\cline { 3 - 6 } & & $20-29$ & $30-39$ & $40-49$ & $>50$ \\
\hline Médico especialista & $\mathrm{F}(\mathrm{n}=24)$ & - & $6(25,0)$ & $11(45,8)$ & $7(29,2)$ \\
$(\mathrm{n}=52)$ & $\mathrm{M}(\mathrm{n}=28)$ & - & $3(10,7)$ & $15(53,6)$ & $10(35,7)$ \\
& Total & - & $9(17,3)$ & $26(50,0)$ & $17(32,7)$ \\
Médico Residente & $\mathrm{F}(\mathrm{n}=52)$ & $26(50,0)$ & $24(46,2)$ & $2(3,8)$ & - \\
$(\mathrm{n}=84)$ & $\mathrm{M}(\mathrm{n}=32)$ & $14(43,8)$ & $16(50,0)$ & $2(6,3)$ & - \\
& Total & $40(47,6)$ & $40(47,6)$ & $4(4,8)$ & - \\
Población general & $\mathrm{F} \mathrm{(n=212)}$ & $98(46,2)$ & $47(22,2)$ & $26(12,3)$ & $41(19,3)$ \\
$(\mathrm{n}=494)$ & $\mathrm{M}(\mathrm{n}=282)$ & $136(48,2)$ & $62(21,9)$ & $27(9,6)$ & $57(20,2)$ \\
& Total & $234(47,4)$ & $109(22,1)$ & $53(10,7)$ & $98(19,8)$ \\
\hline
\end{tabular}

$\mathrm{F}=$ femenino; $\mathrm{M}=$ masculino.

\section{TABLA II}

MÉDICOS TRATANTES DE OTROS MÉDICOS.

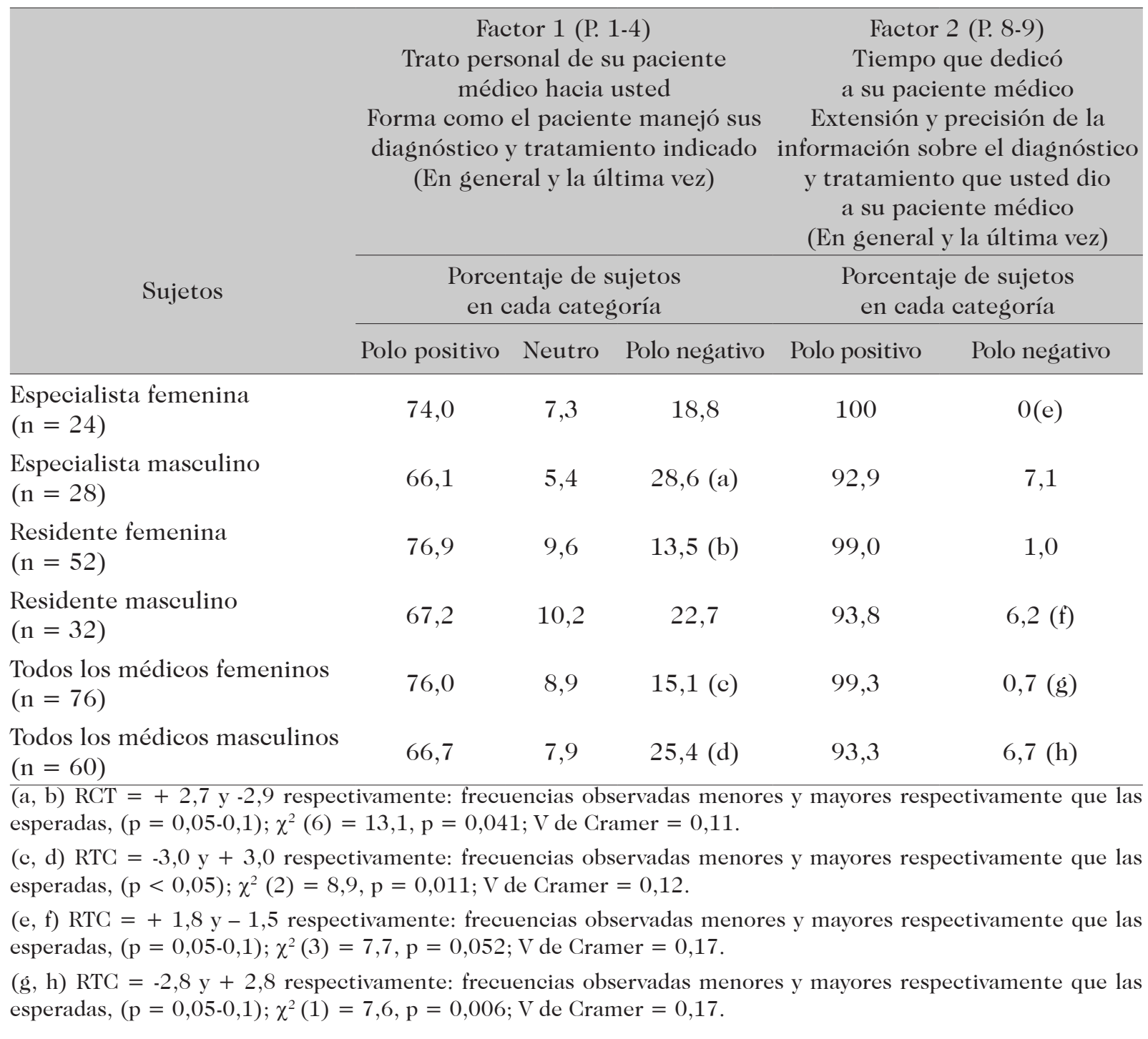


En síntesis, la frecuencia de una opinión negativa en los especialistas masculinos sobre el trato personal y la receptividad sobre los diaǵnósticos y tratamientos ofrecidos por parte de los pacientes médicos fue la más alta de todos los grupos; la frecuencia de opinión negativa en los residentes femeninos fue la más baja.

Factor 2, preguntas 8-9 integradas: Opinión del médico tratante sobre el tiempo que le dedicó a su paciente médico, y sobre las características de la información otorgada sobre el diagnóstico y tratamiento durante la última consulta (Tabla II).

Los especialistas masculinos presentaron la frecuencia más alta de opinión negativa, pero la significación estadística fue marginal $(7,1 \%, p=0,052)$. En conjunto, los médicos masculinos reportaron una mayor frecuencia de opinión negativa que los médicos femeninos $(6,7 \%$ vs. $0,7 \%)$, también con una significación marginal $(\mathrm{p}=$ 0,05-0,1).

Pregunta 10: Interés del médico-paciente en cumplir las indicaciones brindadas.

No se observaron diferencias significativas entre los grupos $(\mathrm{p}>0,05)$. Datos no mostrados.

Pregunta 11-12: Seguimiento del paciente-médico por parte del médico tratante.

Entre el 56 y 64\% de los médicos realizaron un seguimiento del paciente-médico (pregunta 11), sin diferencias significativas entre los grupos sobre la magnitud del contacto $(\mathrm{p}>0,05$, pregunta 12$)$.

Influencia de la especialidad médica, sexo y grado de familiaridad con el médicopaciente, sobre la opinión acerca de la última consulta realizada (preguntas 5-7)

Pregunta 5: influencia de la especialidad del médico-paciente

Opinión sobre la actitud del médico paciente sobre el médico tratante, los diagnnósticos y tratamientos recibidos (factor 1 ).
No se observaron diferencias signnificativas en ninguno de los grupos $(\mathrm{p}>0,05)$.

- Opinión del médico tratante sobre el tiempo que le dedicó a su paciente médico, y sobre las características de la información otorgada sobre el diagnóstico y tratamiento (factor 2).

Los médicos residentes reportaron una actitud más positiva de su parte hacia los médicos-pacientes con especialidad clínica que con especialidad quirúrgica: $96,6 \%$ vs. $66,2 \%$, prueba exacta de Fisher, $\mathrm{p}=0,041$. No se observaron diferencias significativas en los otros grupos de médicos.

- Opinión del médico tratante sobre el interés de su paciente médico en dar continuidad al tratamiento indicado (p. 10), y a mantenerse en contacto (p. 12).

No se detectaron diferencias significativas entre los grupos $(\mathrm{p}>0,05)$.

Pregunta 6: influencia del sexo del médico-paciente

- Opinión sobre la actitud del médico paciente sobre el médico tratante, los diaǵnósticos y tratamientos recibidos (factor 1).

Los especialistas y residentes masculinos reportaron una frecuencia significativamente mayor que los femeninos de actitud negativa por parte del paciente-médico cuando éste era hombre: paciente masculino vs. femenino: especialistas: $46,4 \%$ vs. $10,4 \%$ $(\mathrm{RTC}=4,2, \mathrm{p}<0,05)$; residentes: $37,5 \%$ vs. $3,6 \%(\mathrm{RTC}=4,5, \mathrm{p}<0,05)$. En los médicos femeninos no se observó esta diferencia.

- Opinión del médico tratante sobre el tiempo que le dedicó a su paciente médico, y sobre las características de la información otorgada sobre el diagnóstico y tratamiento (factor 2).

No se encontraron diferencias significativas entre los grupos $(\mathrm{p}>0,05)$. 
- Opinión del médico tratante sobre el interés de su paciente médico en dar continuidad al tratamiento indicado (pregunta 10), y a mantenerse en contacto con el médico tratante (pregunta 12).

No se detectaron diferencias significativas entre los grupos $(\mathrm{p}>0,05)$.

Pregunta 7: influencia de la relación de familiaridad o amistad del médico-paciente con el médico tratante

- Opinión sobre la actitud del médico paciente sobre el médico tratante, los diaǵnósticos y tratamientos recibidos (factor 1).

Los médicos femeninos manifestaron una frecuencia significativamente menor que los masculinos de actitud negativa por parte del médico-paciente cuando éste era familiar o amigo: (relación vs. no relación: especialistas: $0,0 \%$ vs. $30 \%(\mathrm{RTC}=-3,6, \mathrm{p}$ $<0,05)$; residentes: $0,0 \%$ vs. $18,4 \%$ ( $\mathrm{RTC}=$ $-3,5, \mathrm{p}<0,05)$.

En forma narrativa se afirma entonces, que los médicos de sexo femenino informaron una percepción menos negativa de la interacción médico-paciente, cuando tenían vínculos familiares o de amistad con el médico paciente.

- Opinión del médico tratante sobre el tiempo que le dedicó a su paciente médico, y sobre las características de la información otorgada a este sobre el diaǵnóstico y tratamiento (factor 2).

No se encontraron diferencias significativas $(\mathrm{p}>0,05)$.

- Opinión del médico tratante sobre el interés de su paciente médico en dar continuidad al tratamiento indicado ( $\mathrm{p}$. 10), y a mantenerse en contacto con el médico tratante (p. 12).

No se detectaron diferencias significativas entre los grupos $(\mathrm{p}>0,05)$.
Pregunta 13: descripción narrativa de respuestas abiertas

13.1) Aspectos positivos de la actitud del paciente-médico: porcentaje de médicos tratantes que opinaron así:

a. Adecuada comprensión de los diaǵnósticos, $19 \%$.

b. Actitud colaboradora durante la entrevista, $12 \%$.

c. Confianza depositada en el colega tratante, $11 \%$.

13.2) Aspectos negativos de la actitud del paciente-médico: porcentaje de médicos tratantes que opinaron así:

a. Automedicación, $19 \%$.

b. Impaciencia, $13 \%$.

c. Crítica, confrontación, desacuerdo o descalificación de los diaǵnósticos o tratamientos, $13 \%$.

13. 3) ¿Qué piensa el médico tratante que debería cambiar el paciente-médico?

a. Actitud frente a su propia enfermedad, $18 \%$.

b. Cumplimiento del rol de paciente, $16 \%$.

c. Aceptación de que necesita ayuda, $12 \%$.

El médico y la población general como pacientes (preguntas 14-26).

Preguntas 14-17 integradas, factor 3. Opinión sobre el trato brindado por el médico tratante, y sobre los diagnósticos y tratamientos que indicó (en general y en la última consulta) (Tabla III).

Los especialistas femeninos y ambos grupos de médicos masculinos presentaron frecuencias significativamente elevadas de opinión negativa. Los especialistas masculinos manifestaron la frecuencia más elevada. En conjunto, los especialistas reportaron 
TABLA III

LOS MÉDICOS Y LA POBLACIÓN GENERAL COMO PACIENTES.

\begin{tabular}{|c|c|c|c|}
\hline \multirow[b]{2}{*}{ Sujetos } & \multicolumn{3}{|c|}{$\begin{array}{c}\text { Factor } 3 \text { (P. 14-17) } \\
\text { ¿Cómo fue el trato que usted recibió del doctor? } \\
\text { ¿Qué opina del diagnóstico y tratamiento que recibió? } \\
\text { (En general y la última vez) }\end{array}$} \\
\hline & Polo positivo & Neutro & Polo negativo \\
\hline Especialistas femeninos $(\mathrm{n}=24)$ & 68,8 & 5,2 & 26,0 (a) \\
\hline Especialistas masculinos $(\mathrm{n}=28)$ & 50,9 (b) & 10,7 & 38,4 (a) \\
\hline Residentes femeninos $(n=52)$ & 82,7 & 6,2 & 11,1 \\
\hline Residentes masculinos $(\mathrm{n}=32)$ & 69,5 & 8,6 & 21,9 (a) \\
\hline Población general femenina $(\mathrm{n}=212)$ & 81,9 (a) & 7,7 (b) & 10,4 \\
\hline Población general masculina $(\mathrm{n}=282)$ & 79,5 & 13,9 (a) & 6,6 (b) \\
\hline
\end{tabular}

una frecuencia mayor de opinión negativa que los residentes, pero la significación fue marginal: $38,5 \%$ vs $21,4 \%, \mathrm{p}=0,062$.

En la PG describimos una opinión con resultados significativos: la femenina, obtuvo frecuencias observadas de opinión positiva mayores que las esperadas, y la masculina, frecuencias observadas de opinión negativa menores que las esperadas ( $\mathrm{p}<0,05)$.

En conjunto, todos los médicos tienen una opinión más negativa que la $\mathrm{PG}$, en particular los especialistas masculinos.

El análisis de correlación entre la edad y la puntuación en el factor 3 demostró una correlación significativa negativa en los residentes femeninos (a menor edad, peor opinión). La PG femenina presentó una correlación significativa positiva, es decir, a mayor edad, peor opinión (puntuación más elevada) (Tabla IV).

Preguntas 21-23, Factor 4: Tiempo que le dedicó el médico, información brindada sobre el diagnóstico, tratamiento y seguimiento de su caso, durante su última consulta (Tabla V).

Los especialistas masculinos presentaron la frecuencia más alta de opinión nega- tiva, que se reflejó en una alta signnificación estadística $(\mathrm{p}<0,001)$. En conjunto, todos los especialistas reportaron una frecuencia mayor de opinión negativa que todos los residentes, pero la significación fue marginal: $32,7 \%$ vs $19,0 \%, \mathrm{p}=0,07$.

Preguntas 24 y 25. Posibilidad de contactar al médico tratante

No se encontraron diferencias significativas entre los grupos. Sin embargo, dado que se observaron unas tendencias congruentes con los resultados anteriores, los valores se presentan en la Tabla VI. La frecuencia con la que pudo ser contactado el médico tratante no se reporta en la $\mathrm{PG}$, pues las respuestas fueron equívocas (pregunta 25).

Los especialistas femeninos y masculinos manifestaron las frecuencias más altas de descontento sobre la posibilidad de contactar al médico tratante (pregunta 25).

Influencia de la especialidad médica ( $p$. 18), sexo (p. 19) y tipo de relación del médico tratante (p. 20) con el médico paciente sobre la opinión acerca de la última consulta a la que acudió 
TABLA IV

MATRIZ DE CORRELACIÓN ENTRE EDAD Y LA OPINIÓN DE LOS GRUPOS COMO PACIENTES (FACTORES 3 Y 4).

\begin{tabular}{|c|c|c|}
\hline Grupo & Factor 3: $r(n) p$ & Factor 4: $r(n) p$ \\
\hline Especialistas femeninos $(n=24)$ & $0,032(96) 0,73$ & $0,007(72) 0,9$ \\
\hline Especialistas masculinos $(n=28)$ & 0,092 (112) 0,29 & $-0,09(84) 0,4$ \\
\hline Residentes femeninos $(\mathrm{n}=52)$ & $-0,18(208) 0,007$ & $-0,29(156)<0,001$ \\
\hline Residentes masculinos $(\mathrm{n}=32)$ & 0,015 (128) 0,86 & $0,1(96) 0,3$ \\
\hline Población general femenina $(n=212)$ & $0,16(846)<0,001$ & $-0,024(634) 0,5$ \\
\hline Población general masculina $(\mathrm{n}=282)$ & $0,044(1128) 0,10$ & $0,005(846) 0,9$ \\
\hline
\end{tabular}

$\mathrm{r}=$ coeficiente de correlación de Kendall-Tau; a mayor puntuación en la escala, más negativa la opinión; $\mathrm{n}=$ número de sujetos por grupo; $\mathrm{p}=$ probabilidad asociada al análisis de correlación. El número de sujetos por grupo es mayor que el número real porque el factor 3 incluye 4 preguntas (14-17) y el factor 4 incluye 3 preguntas. Los resultados significativos se resaltan en negritas.

TABLA V

LOS MÉDICOS Y LA POBLACIÓN GENERAL COMO PACIENTES.

\begin{tabular}{|c|c|c|}
\hline \multirow[b]{2}{*}{ Sujetos } & \multicolumn{2}{|c|}{$\begin{array}{c}\text { Factor } 4 \text { (P. 21-23) } \\
\text { Tiempo que le dedicó el médico, información brindada sobre } \\
\text { el diagnóstico, tratamiento y seguimiento de su caso } \\
\text { (La última consulta) }\end{array}$} \\
\hline & Polo positivo & Polo negativo \\
\hline Especialistas femeninos $(\mathrm{n}=24)$ & 75,0 & 25,0 \\
\hline Especialistas masculinos $(\mathrm{n}=28)$ & $57,1(b)$ & 42,9 (a) \\
\hline Residentes femeninos $(n=52)$ & 82,1 & 17,9 \\
\hline Residentes masculinos $(\mathrm{n}=32)$ & 72,9 & 21,7 \\
\hline Población general femenina $(\mathrm{n}=212)$ & 76,4 & 23,6 \\
\hline Población general masculina $(\mathrm{n}=282)$ & 79,7 & 20,3 \\
\hline
\end{tabular}

$\chi^{2}(5)=25,4, p<0,001 ; \mathrm{V}$ de Cramer $=0,12 ;$ (a) $\mathrm{RTC}=4,5$, frecuencia observada significativamente mayor que las esperada; (b) RTC $=-4,54$, frecuencia observada significativamente menor que la esperada.

TABLA VI

FRECUENCIA Y CALIDAD DE CONTACTO CON EL MÉDICO TRATANTE.

\begin{tabular}{lcc}
\hline \multicolumn{1}{c}{ Sujetos } & $\begin{array}{c}\text { Porcentaje que pudo } \\
\text { contactar al médico } \\
\text { tratante (P. 24) }\end{array}$ & $\begin{array}{c}\text { Porcentaje de opinión negativa } \\
\text { con la frecuencia de } \\
\text { contacto (P. 25) }\end{array}$ \\
\hline Especialistas femeninos $(\mathrm{n}=24)$ & 91,7 & 31,8 \\
Especialistas masculinos $(\mathrm{n}=28)$ & 75 & 38,1 \\
Residentes femeninos $(\mathrm{n}=52)$ & 78,8 & 12,2 \\
Residentes masculinos $(\mathrm{n}=32)$ & 68,8 & 13,6 \\
Población general femenina $(\mathrm{n}=212)$ & 75,2 & - \\
Población general masculina $(\mathrm{n}=282)$ & 74,4 & - \\
\hline
\end{tabular}

Ninguna de las comparaciones alcanzó signnificación estadística, p > 0,05. 
Pregunta 18: influencia de la especialidad del médico tratante

- Opinión sobre el trato brindado por el médico tratante, y sobre los diagnósticos y tratamientos que indicó (en la última consulta) (Factor 3).

No se encontraron diferencias significativas entre los grupos $(\mathrm{p}>0,05)$.

- Opinión sobre el tiempo que le dedicó el médico, información brindada sobre el diagnóstico, tratamiento y seguimiento de su caso, durante su última consulta (Factor 4).

No se encontraron diferencias significativas entre los grupos ( $p>0,05)$.

- Opinión sobre el contacto o seguimiento de su caso con el médico tratante (preguntas 24 y 25)

No se encontraron diferencias significativas entre los grupos de médicos $(\mathrm{p}>0,05)$.

Pregunta 19: influencia del sexo del médico tratante

- Opinión sobre el trato brindado por el médico tratante, y sobre los diagnósticos y tratamientos que indicó (en la última consulta) (Factor 3).

No se encontraron diferencias significativas entre los grupos ( $\mathrm{p}>0,05)$.

- Opinión sobre el tiempo que le dedicó el médico, información brindada sobre el diaǵnóstico, tratamiento y seguimiento de su caso, durante su última consulta (Factor 4).

Los residentes masculinos reportaron una frecuencia elevada de opinión negativa cuando el médico tratante era hombre: $36,7 \%$ vs. $11,1 \%$. La significación estadística fue marginal: $\mathrm{RCT}=2,7, \mathrm{p}=0,05-0,1$. No se reportaron diferencias en los otros grupos de médicos.
- Opinión sobre el contacto o seguimiento de su caso con el médico tratante (preguntas 24 y 25)

No se detectaron diferencias significativas entre los grupos, sin embargo, los especialistas masculinos reportaron la frecuencia más elevada de opinión negativa sobre la magnitud de contacto con su médico tratante $(50 \%$, RCT $=2, p>0,1)$.

Pregunta 20: influencia de la relación de familiaridad o amistad del médico-paciente con el médico tratante

- Opinión sobre el trato brindado por el médico tratante, y sobre los diagnósticos y tratamientos que indicó (en la última consulta) (Factor 3).

No se encontraron diferencias significativas entre los 4 grupos de médicos ( $p>$ $0,05)$.

- Opinión sobre el tiempo que le dedicó el médico, información brindada sobre el diagnóstico, tratamiento y seguimiento de su caso, durante su última consulta (Factor 4).

Los residentes masculinos reportaron una frecuencia elevada de opinión negativa cuando el médico tratante tenía vínculos de amistad o parentesco con ellos: $37,7 \%$ vs. $11,9 \%$ (no vínculos). La significación estadística fue marginal: $\mathrm{RCT}=2,6, \mathrm{p}=0,05$ 0,1 . No se reportaron diferencias en los otros grupos de médicos.

- Opinión sobre el contacto o seguimiento de su caso con el médico tratante (preguntas 24 y 25)

No se detectaron diferencias significativas entre los grupos, sin embargo, los especialistas masculinos reportaron la mas alta frecuencia de opinión negativa cuando el médico tratante tenia vínculos de amistad o familiaridad. Sin embargo, no se alcanzó significación estadística (50\%, RCT $=2,2, \mathrm{p}$ $>0,1)$. 
Pregunta 26: descripción narrativa de respuestas abiertas

26.1) Aspectos positivos de la actitud del médico tratante: porcentaje de sujetos que opinaron así:

a. Calidad en la interacción personal: buen trato, $16 \%$.

b. Atención esmerada: cuidado, consuelo, interés y dedicación proporcionada al colega paciente, $13 \%$.

c. Precisión en los diagnnósticos, $10 \%$.

26.2) Aspectos negativos de la actitud del médico tratante: porcentaje de sujetos que opinaron así:
a. Ninguno, $14 \%$.
b. Apatía o indiferencia, $10 \%$.
c. Atención superficial, $10 \%$.

26. 3. ¿Qué piensa el paciente que debería cambiar el médico tratante?

a. Humanización de la relación

médico paciente, $18 \%$.

b. Descartar la condición de médico y asegurar anamnesis y examen físico completo, aumentar el tiempo de atención y mejorar la comunicación, $10 \%$.

\section{DISCUSIÓN}

Se confirmó la hipótesis propuesta: una proporción significativa de médicos manifestó su descontento como tratante de otro galeno y como paciente. Además, el sexo y la edad fueron variables que influyeron en los resultados observados. Esta hipótesis se formuló a partir de anécdotas, y como tal, no parte de antecedentes sistematizados. La hipótesis se enunció de manera unidireccional hacia la opinión negativa de los médicos como tratantes de otros médicos y como pacientes, y del mismo modo se orientó el análisis estadístico.
Los especialistas masculinos presentaron la mayor frecuencia de opinión negativa al tratar a otro colega, y las residentes femeninas, la menor frecuencia. En conjunto, todos los médicos masculinos reportaron una frecuencia mayor de opinión negativa en los dos factores que exploraron su papel como médico tratante de otro colega. La opinión de los especialistas masculinos se correlacionó positivamente con la edad, es decir, a mayor edad, más negativa la percepción.

El análisis de otras variables intervinientes en la opinión del médico cuando trató otro médico también arrojó resultados de interés:

a. Los residentes en conjunto reportaron una actitud más negativa de su parte hacia el médico-paciente de especialidad quirúrgica, en comparación a los de especialidad clínica

b. Los especialistas y residentes masculinos reportaron una frecuencia significativamente mayor de actitud negativa por parte del paciente-médico cuando éste era hombre.

c. Los médicos de sexo femenino informaron una percepción menos negativa de la interacción médicopaciente, cuando el médico-paciente tenía vínculos familiares o de amistad con el médico tratante

d. Los médicos entrevistados manifestaron narrativamente que el médico debe asumir adecuadamente su papel de paciente.

La opinión de los médicos y de la PG en su papel de pacientes fue consistente con los resultados anteriores: los especialistas masculinos presentaron las frecuencias negativas más elevadas Los residentes femeninos presentaron frecuencias más bajas de insatisfacción en su condición de pacientes. En conjunto, los médicos femeninos presentaron frecuencias más bajas de opinión ne- 
gativa, al iǵual que todos los residentes en comparación a todos los especialistas. Los sujetos de la PG reportaron frecuencias de opinión negativa menores que los médicos, en particular los sujetos masculinos. Los especialistas femeninos y masculinos manifestaron las frecuencias más altas de descontento sobre la posibilidad de contactar al médico tratante.

Con relación a otras variables intervinientes, se obtuvieron los siguientes resultados en la evaluación de los médicos y la PG como pacientes:

a. Los residentes masculinos reportaron una frecuencia elevada de opinión negativa cuando el médico tratante era del sexo masculino

b. Los especialistas masculinos reportaron la frecuencia más elevada de opinión negativa sobre la maǵnitud de contacto con su médico tratante

c. Los médicos masculinos, tanto residentes como especialistas reportaron las frecuencias más altas de opinión negativa del seguimiento y contacto con el médico tratante, cuando este era del sexo masculino.

d. En forma narrativa los médicos como pacientes enfatizaron que cuando trate a un colega, el médico debe "dejar de lado" la condición profesional del paciente.

No encontramos estudios publicados que evalúen simultáneamente y con un mismo instrumento la PG y los médicos. Es por ello por lo que no pudimos discutir nuestros resultados en el contexto de otras investigaciones relacionadas.

Mihailescu y Neiterman (11), enfatizaron la dificultad en comparar los estudios realizados en los Estados Unidos y Canadá con aquellos conducidos en América Latina.
Hamui-Sutton y col., en el 2013 evaluaron la satisfacción con la atención médica recibida en 1.457 .964 sujetos de la $\mathrm{PG}$ mexicana (64,5\% mujeres), y encontraron los siguientes resultados: muy satisfecho $(37,3 \%)$; satisfecho $(54,3 \%)$; poco satisfecho $(7,5 \%)$ y nada satisfecho $(0,6)(12)$. El estudio mexicano y el nuestro, coinciden en que la PG tiene un alto nivel de satisfacción con la atención sanitaria. En Venezuela, la calidad de la atención disminuyó después del año 2015. Nuestro estudio tiene la fortaleza de haber evaluado simultáneamente todos los grupos, de modo que se garantizó la comparabilidad entre ellos. Estos resultados pueden ser relevantes para otros países hispanos donde la atención sanitaria institucional, y mediante aseguradoras privadas, puede haber sido impactada por los recientes eventos socio políticos y por la epidemia mundial de COVID-19.

La metodología utilizada no permite inferir las razones del descontento del especialista masculino. Seguramente se trata de un conjunto complejo de factores del médicopaciente y del médico-tratante.

Un estudio en 20.901 sujetos de la PG de los Estados Unidos encontró un mayor nivel de satisfacción con la atención recibida en sujetos mayores de 65 años comparado con sujetos de menor edad; es este estudio no se detectó una influencia signnificativa del sexo del usuario (13). Nuestros resultados con los médicos especialistas van en dirección contraria.

En síntesis, los especialistas masculinos se comportan en forma opuesta a la tendencia observada en la PG de otros países, en la cual, a mayor edad, mayor satisfacción con la atención médica recibida.

No se exploró la personalidad en los sujetos entrevistados, de modo que discutiremos este aspecto a fin de plantear hipótesis para futuros estudios.

Stienen y col. (14), realizaron un estudio multicéntrico en Europa y Canadá, en donde exploraron el perfil de personalidad en 2.345 médicos mediante los Cinco Facto-

Vol. 62(4): 339 - 356, 2021 
res de Personalidad. Al comparar cirujanos y clínicos, los eirujanos presentaron puntuaciones mayores de extroversión $(\mathrm{p}=0,003)$ y apertura a la experiencia $(\mathrm{p}=0,002)$ y niveles más bajos de neuroticismo $(\mathrm{p}<0,001)$.

En el contexto psicológico el cirujano, en comparación al clínico, sería más "extrovertido" (asertivo, gregario, con emociones positivas), "abierto a la experiencia" (fantasía, sentimientos, acciones) y con menos "neuroticismo" (hostilidad, depresión, ansiedad social, impulsividad, vulnerabilidad). Podríamos especular que el clínico tendería a ser menos asertivo, más vulnerable y reservado que el cirujano, ante la tensión que ocurriese entre un médico y su paciente, y tener así una opinión negativa de la interacción. Los hombres con rasgos obsesivos y evasivos (elevado neuroticismo) presentan también mayor riesgo suicida en edades avanzadas (15). Futuros estudios deben explorar este importante aspecto.

El médico y sus familiares pueden esperar que un galeno consultante sea especialmente amable y cuidadoso con ellos, y además ser exonerados de los honorarios profesionales; se entiende entonces que se desarrolle tensión entre las partes involucradas. En el peor de los casos, el médico-paciente puede opinar que no recibió la atención que merecían; por su parte, el médico tratante puede considerar que no se valoró adecuadamente su labor.

No encontramos estudios publicados relacionados al nuestro, que aborden el delicado tema de la remuneración (o su ausencia) al médico tratante. Podemos predecir que el aspecto económico difiere signnificativamente en comunidades que cuentan con seguros médicos eficientes, tanto públicos o privados, en comparación con lo que ocurre actualmente en países como el nuestro en donde el sistema de seguros no alcanza a la mayoría de la población.

En una publicación preliminar que no incluía los datos de la PG, se presentó la opinión de las parejas de los médicos y los resultados fueron consistentes con los de los médicos: las parejas de los especialistas masculinos reportaron frecuencias elevadas de descontento con la atención médica recibida (16). Decidimos excluir estos resultados porque el número de especialistas que respondió la encuesta es distinto al número de parejas que lo hizo, y no podemos parear cada médico con su pareja por no haber verificado esto en la recepción y transcripción de los datos. Tal falta de apareamiento dificulta el análisis estadístico inferencial. Este resultado consistente con lo reportado aquí debe tomarse en cuenta en estudios posteriores.

Un resultado consistente a lo largo de todos los análisis fue que los especialistas masculinos presentaron los porcentajes más elevados de insatisfacción, tanto como usuarios como de proveedores de atención médica. Estos resultados esperan su replicación en estudios multicéntricos, con muestras de mayor tamaño y control de las características demográficas, psicológicas y profesionales. Deben diseñarse estudios que además evalúen variables objetivas tales como el curso de la enfermedad, sus complicaciones, tipo de tratamiento indicado -clínico o quirúrgico-, la opinión de los cuidadores o familiares y otras que puedan contrastarse con las opiniones subjetivas del usuario y del profesional que brinda la atención médica.

Este estudio tiene varias limitaciones:

1. No se encontraron trabajos publicados que incluyan en forma concurrente los médicos y la PG con los cuales comparar nuestros resultados.

2. La opinión de los entrevistados tuvo una distribución de frecuencias similar cuando se refirió a su experiencia "en general" y "en la última consulta". No sabemos si la similitud de respuesta con relación al marco cronológico obedece a un "sesgo del recuerdo". Esto debe abordarse en investigaciones posteriores. Sin embargo, dado que sí encontramos diferencias individuales en las respuestas, consideramos cada pregunta como un ítem específico dentro 
de los diversos factores. A fin de atenuar el error alfa, utilizamos las correcciones de continuidad y de Bonferroni.

3. No se diferenció si la atención médica fue realizada en instituciones públicas o privadas, si bien todos los médicos entrevistados trabajaban en un hospital universitario público. Finalmente, el procesamiento de la información de la sección de respuestas narrativas de los sujetos como pacientes, no discriminó entre médicos y sujetos de la PG. El presente estudio puede entonces considerarse como una investigación preliminar que provee una extensa base de resultados que esperan replicación y comparaciones pertinentes.

Se recomienda difundir y estimular esta línea de investigación, y desarrollar programas ad hoc para cada entorno particular, con el fin de optimizar la atención de los médicos y su familia, al mismo tiempo que se respeta y reconoce la actividad de los médicos tratantes. Estos resultados pueden ser más relevantes aún en la presente época afectada por la pandemia de COVID-19.

\section{AGRADECIMIENTO}

Los autores agradecen la entusiasta colaboración de los médicos del I.H.U.L.A, y las personas de la población general.

\section{REFERENCIAS}

1. Albujar-Baca PF. Cuando el médico es el paciente. Acta Med Per 2015; 32 (3):164.

2. Rogers T. Barriers to the doctor as patient role. A cultural construct. Aust Fam Physician 1998; 27 : 1009-10013.

3. Bachman JW. The problem with patient satisfaction scores. Fam Pract Manag 2016; Jan/Feb: 23-27.

4. Panagioti M, Geraghty K, Johnson J, Panagopoulou E, Chew-Graham C, Peters D, Hodkinson A, Riley R, Esmail A. Association between physician burnout and patient safety, professionalism, and patient satisfaction: A systematic review and meta-analysis.
JAMA Intern Med. 2018; 178: 1317-1330. doi: 10.1001/jamainternmed.2018.3713.

5. Rothenberger DA. Physician burnout and well-being: A systematic review and framework for action. Dis Colon Rectum 2017; 60:567-576. doi: 10.1097/DCR.0000 000000000844 .

6. Dutheil F, Aubert C, Pereira B, Dambrun M, Moustafa F, Mermillod M, Baker JS, Trousselard M, Lesage FX, Nave V. Suicide among physicians and health-care workers: A systematic review and metaanalysis. PLoS ONE. 2019; 14(12): e0226361. doi. org/10.1371/journal. pone.0226361.

7. Baptista T. Acreditación como médico especialista: Guías básicas para elaborar el proyecto de tesis. Universidad de Los Andes Vicerrectorado Académico CODEPRE En coedición con Organon Venezolana S.A, Mérida, Venezuela, 2006.

8. García-García JA, Reding-Bernal A, Lopez-Alvarenga JC. Cálculo del tamaño de la muestra en investigación en educación médica. Inv Ed Med 2013; 2 (8):217-224.

9. www.es.surveymonkey.com/ $/ \mathrm{mp} /$

10. Hernández-Nieto R. Instrumentos de recolección de datos. Validez y Confiabilidad. Normas y Formatos. Mérida, Venezuela: Consejo de Estudios de Postgrado, Universidad de Los Andes. 2011. Disponible en https://www.amazon.com.mx/INSTRUMENTOS-RECOLECGION-DATOS-RafaelHernandez- Nieto-ebook/dp/B007TB4BEE.

11. Mihailescu M, Neiterman E. A scoping review of the literature on the current mental health status of physicians and physicians-in-training in North America. BMC Public Health 2019; 19:1363. https://doi. org/10.1186/s12889-019-7661-9.

12. Hamui Sutton L, García RF, Hernández RA, Ramírez de la Roche OF. Expectativas y experiencias de los usuarios del Sistema de Salud en México: Un estudio de satisfacción con la atención médica. 2013 D.R. Universidad Nacional Autónoma de México, Facultad de Medicina, México. 208 pp.

13. Meg C, Kong MC, Camacho FT, Feldman SR, Anderson RT, Balkrishnan R. Correlates of patient satisfaction with physician visit: differences between elderly and non-elderly survey respondents. Health Qual Life Outc 2007; 5:62. doi: 10.1186/1477-7525-5-62. 
14. Stienen MN, Scholtes F, Samuel R, Weil A, Weyerbrock A, Surbeck A. Different but similar: personality traits of surgeons and internists-results of a cross-sectional observational study. BMJ Open. 2018; 8: e021310. doi:10.1136/bmjopen-2017-021310 1.

15. Szücs A, Szanto K, Aubry JM, Dombrovski AY. Personality and suicidal behavior in old age: a systematic literature review. Front. Psychiatry 2018; 9: 128. doi: 10.3389/fpsyt.2018.00128.
16. Delgado H, Salcedo R, Uzcátegui E, Paez $\mathrm{X}$, Baptista T. Percepción de los médicos y sus parejas sobre el trato recibido durante la consulta con otro facultativo en Mérida, Venezuela. Archivos Venezolanos de Neurología y psiquiatría. 2013; 59 (Enero-Junio), 19-26. 